\title{
Imaging of surface plasmon polariton interference using phase-sensitive photon scanning tunneling microscope
}

\author{
J. Jose · F.B. Segerink • J.P. Korterik · J.L. Herek • \\ H.L. Offerhaus
}

Received: 8 January 2010 / Accepted: 3 December 2010 / Published online: 6 January 2011

(c) The Author(s) 2011. This article is published with open access at Springerlink.com

\begin{abstract}
We report the surface plasmon polariton interference, generated via a 'buried' gold grating, and imaged using a phase-sensitive Photon Scanning Tunneling Microscope (PSTM). The phase-resolved PSTM measurement unravels the complex surface plasmon polariton interference fields at the gold-air interface.
\end{abstract}

\section{Introduction}

One prominent development in the field of optical microscopy date back to 1989 , when Reddick et al. [1] developed a new form of scanning optical microscope called Photon Scanning Tunneling Microscope (PSTM). The operating principle of PSTM is based on the generation of evanescent waves by total internal reflection of a light beam followed by its frustration using a sharpened optical fiber probe. Experimentally verified in 1957 [2], Surface Plasmon Polaritons (SPPs) excited at a metal-dielectric interface were found to inherit many properties of the evanescent waves, except that the fields associated with the SPPs decays exponentially into both sides of the metal-dielectric interface. SPPs are charge density waves that can be optically excited on the metal-dielectric interface when the inplane wave vector component of the incident photon $\left(k_{x}\right)$ matches the SPP wave vector $\left(k_{s p}\right)$ [3]. Excitation of multiple SPPs by patterning the metal surface have found a range of applications like surface plasmon interference nanolithography [4], photonic band gap materials [5], and sensing devices [6]. However, there has not been an attempt made to

J. Jose $(\bowtie) \cdot$ F.B. Segerink · J.P. Korterik · J.L. Herek

H.L. Offerhaus

Mesa+ Institute for Nanotechnology, Optical Sciences Group, University of Twente, $7500 \mathrm{AE}$, Enschede, The Netherlands

e-mail: j.jose@tnw.utwente.nl separate the different SPPs excited on a patterned metal surface.

A conventional PSTM measurement [7, 8] on a patterned metal surface yields only the intensity of the optical field. In order to separate the multiple SPPs, one should measure both amplitude and phase of the optical field on the metal surface. In this work, we use a heterodyne interferometric (or phase-sensitive) PSTM [9-13] to measure the complex SPP interference field, generated on a gold-air interface by simultaneously exciting two SPP waves on the interface: one using prism coupling and another using grating coupling [3]. The interference between the two SPP waves, having same energy and propagating in different in-plane directions, manifests as a beating pattern formed along the direction of $k_{x}$. The two SPP modes are separated by filtering the desired wave vectors in the two-dimensional Fourier Transform image of the total optical field [11].

The paper is organized as follows. Section 2 explains the different steps involved in the fabrication of a gold buried grating. In Sect. 3, we explain the phase matching condition for the simultaneous excitation of two SPPs and the experimental procedure to acquire the phase-sensitive PSTM images of the SPP interference. The PSTM images showing the interference of two SPPs along with discussions and conclusion are presented in Sects. 4 and 5, respectively.

\section{Fabrication}

In a Kretschmann-Raether (KR) configuration [14] for the excitation of the SPPs, a periodic corrugation of the metalair interface scatters the SPPs at that interface into radiation. The scattering can be minimized by turning the grating upside down to form a "buried grating." The glassmetal interface is corrugated leaving a flat metal layer on 
Fig. 1 Schematic of the fabrication of a gold buried grating

Fig. 2 (a) The reciprocal space representation of the theoretical phase matching condition to excite two SPPs by the in-plane rotation of the grating wave vector $k_{g}$. The solid red curve denotes the momentum of the SPPs at the gold-air interface, the solid red arrow denotes the wave vector of the SPPs, the dashed black arrow denotes the wave vector of the +1 st diffracted order, and the solid green arrows indicate the grating wave vectors. (b) Schematic of a heterodyne interferometric PSTM

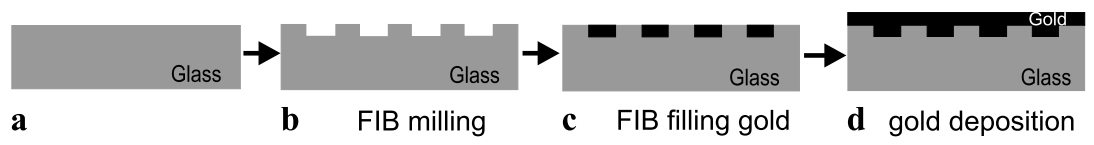

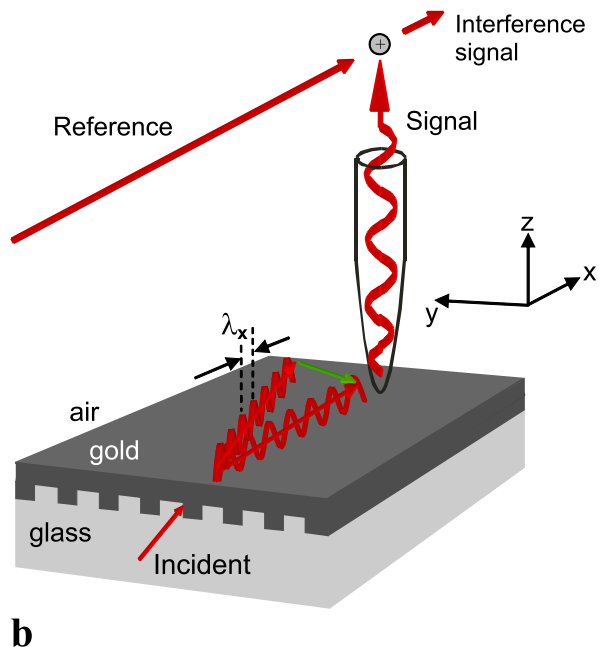

top to support SPPs [15]. The steps to fabricate a buried gold grating are illustrated in Fig. 1. A 0.5 duty cycle linear grating, with period of $1.65 \mu \mathrm{m}$ and depth of $50 \mathrm{~nm}$, was milled into a $0.15 \mathrm{~mm}$ thick glass cover slip using a Focused Ion Beam (FIB) (see Fig. 1(b)). A thin layer of gold-palladium mixture was deposited on the cover slip prior to the milling action to eliminate charging effects (not shown). The grooves were then filled with gold using the FIB together with a gold gas injection system (see Fig. 1(c)). A $50 \mathrm{~nm}$ thick gold layer was deposited on top of the grating using electron beam evaporation technique (see Fig. 1(d)). A drift of the FIB or an inaccurate dwell time for the deposition of gold caused a FIB positioning error, and hence an over/under filling of the grooves.

\section{Experimental procedure}

The buried gold grating was placed on a glass (BK7) hemispherical prism with index matching oil in between. The hemispherical prism was mounted on a convenient rotating stage providing an azimuthal rotation angle ranging from $0^{\circ}$ to $90^{\circ}$, in steps of $0.5^{\circ}$. A fiber collimator, mounted on a goniometric stage for angles ranging from $42^{\circ}$ to $50.8^{\circ}$, illuminated the sample from the prism side [13]. A polarizer ensured an input beam polarized in a direction parallel (p-polarized) to the plane of incidence. Figure 2(a) shows a reciprocal space representation of the phase matching condition to excite two SPPs at the goldair interface. The incident light coupled to SPPs when $k_{x}$ is equal to $k_{s p}$. The grating wave vector $k_{g}$ was rotated in-plane by an angle $\beta$ such that the -1 st evanescent diffracted order coupled to SPPs. The evanescent waves associated with the 0th and the -1 st diffracted order cannot couple to SPPs at the periodic glass-gold interface due to the higher value of the wave vector SPPs at that interface [3]. Hence, the coupling between the SPPs through the gold film is not relevant in this particular study.

A schematic of the phase-sensitive PSTM for imaging two SPPs is shown in Fig. 2(b). The laser light (free space wavelength of $657.3 \mathrm{~nm}$ ) was split into two the two arms of an interferometer: one formed the reference arm of the interferometer and the other formed the signal arm that illuminated the sample. The optical frequency in the reference arm was shifted by $100 \mathrm{kHz}$ using two acousto-optic modulators (not shown). A metal-coated optical fiber probe was raster scanned on the gold surface. The optical signal, picked up by the fiber probe, was combined with the signal in the reference branch in a $2 \times 2$ fiber coupler (heterodyne mixing). The interference signal from the two outputs of the fiber coupler was $180^{\circ}$ out of phase with each other. A balanced detection scheme [16] was used to cancel the noise in the reference signal and enhance the intensity of the difference signal. The signal was measured using a photodiode and a dual-phase lock-in-amplifier to extract the amplitude and the phase of the optical field on the sample surface. The PSTM was operated in constant distance mode using tuning fork shear-force feedback [17]. Thus, topographical information and complex optical field on the gold surface were simultaneously retrieved. 


\section{Measurements and discussion}

A phase-sensitive PSTM measurement on the buried gold grating is presented in Fig. 3. The topography of the grating shows a residual modulation of $34.5 \pm 3.9 \mathrm{~nm}$ on the surface which is attributed to an over-filling of the grating grooves. A comparison between the topography and the optical amplitude (see Fig. 3(b)) images shows that the optical amplitude is minimum where the topography shows a maximum and vice versa. The former behavior can be attributed to an over-filling of the grooves, mentioned in Sect. 2, which leads to an under-coupling of the prism-coupled SPPs. The minimum in the topography image corresponds to the optimum thickness $(50 \mathrm{~nm})$ to excite prism-coupled SPPs [3], and hence we see a maximum in the optical amplitude. In the real part of the total optical field, shown in Fig. 3(c), a plane wave whose wave vector lies in the plane of incidence is seen. In addition, a modulation of the wave fronts is also visible.

In order to separate the different plane waves present in the total optical field, a Fourier transform of the total optical field is taken. A zoom-in region of the magnitude of the Fourier transform of the total optical field is shown in Fig. 3(d). There is an intense feature in the image which corresponds to the 0th order evanescent wave. The features corresponding to the evanescent diffracted orders are seen on either side of the 0th order feature. The feature corresponding to the +1 st diffracted order is hardly visible in the image. The 0th, -1 st and the +1 st diffracted orders are individually selected from the 2D FT image shown in Fig. 3(d) and shown separately in Figs. 4(a)-4(c). Investigating those components by Fourier back transformation gives three plane waves which propagate at different angles. The angle between the plane waves shown in Figs. 4(a) and 4(b) is $19.3^{\circ}$. That means the two SPPs, coupled individually by the prism and the grating, are at an angle of $19.3^{\circ}$ with respect to each other. The +1 st evanescent diffracted order has a shorter wavelength, as expected from the phase matching diagram shown in Fig. 2(a).

\section{Conclusion}

In conclusion, the complex SPP interference generated at a gold-air interface using a gold buried grating has been measured with sub-wavelength resolution using a phasesensitive PSTM. Fourier analysis untangles the interfering fields and reveals the excitation of two SPPs propagating at different angles. We believe that the capability of a phase-sensitive PSTM to separate the SPPs will find application in the optical characterization of other plasmonic (nano)structures with sub-wavelength resolution.
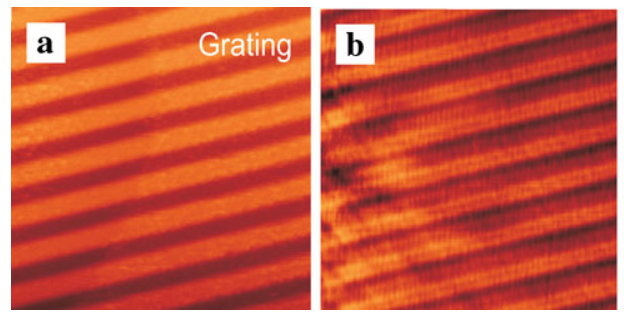

Fig. 3 PSTM measurement of a gold buried grating for a scan range of $13.3 \times 18.5 \mu \mathrm{m}$. (a) The measured topography with dark regions correspond to valleys and brighter regions to peaks, (b) the measured optical amplitude on the gold surface, and (c) the measured optical am-

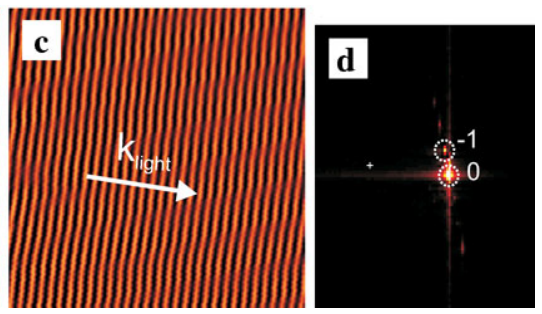

plitude times cosine of the phase of the optical field. The white arrow indicates the propagation direction of light. (d) The magnitude of the Fourier transform of the total optical field with an area of $4.6 \times 6.4 \mu \mathrm{m}$. The white cross indicates the zero-frequency point in the Fourier image

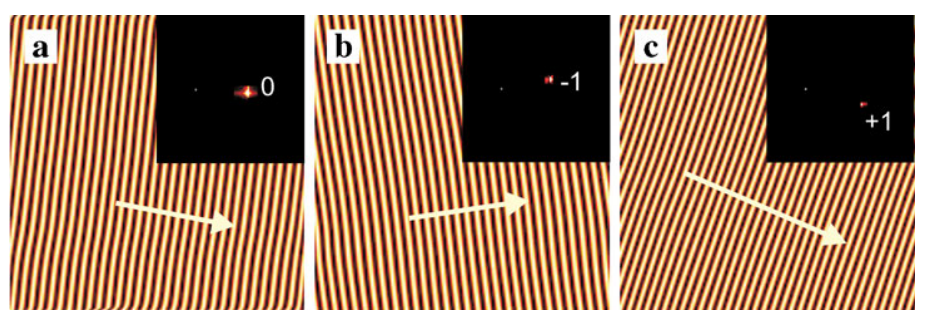

Fig. 4 The Fourier transformation analysis of the optical field excited using a gold buried grating for a scan range of $13.3 \times 18.5 \mu \mathrm{m}$. The insets show the Fourier transform of the different orders filtered from Fig. 3(d) with an area of $4.6 \times 6.4 \mu \mathrm{m}$. The white cross indicates the zero-frequency point in the Fourier image. The Fourier back transform to retrieve the wavelength component of the SPPs coupled by (a) the 0 th order evanescent wave, and (b) the -1 st order evanescent diffracted order. (c) The wavelength component of the +1 st diffracted order having a higher spatial frequency. The cosine of the optical phase is shown. The arrows indicate the propagation direction of the plane waves 
Acknowledgement The authors thank Professor Kobus Kuipers for his valuable comments. This work is supported by NanoNed, a nanotechnology program of the Dutch Ministry of Economic Affairs.

Open Access This article is distributed under the terms of the Creative Commons Attribution Noncommercial License which permits any noncommercial use, distribution, and reproduction in any medium, provided the original author(s) and source are credited.

\section{References}

1. R.C. Reddick, R.J. Warmack, T.L. Ferrell, New form of scanning optical microscopy. Phys. Rev. B 39(1), 767-770 (1989)

2. R.H. Ritchie, Plasma losses by fast electrons in thin films. Phys. Rev. 1(5), 874-881 (1957)

3. H. Raether, Surface Plasmons on Smooth and Rough Surfaces and on Grating (Springer, Berlin, 1988)

4. Z.W. Liu, Q.H. Wei, X. Zhang, Surface plasmon interference nanolithography. Nano Lett. 5(5), 957-961 (2005)

5. S.C. Kitson, W.L. Barnes, J.R. Sambles, Full photonic band gap for surface modes in the visible. Phys. Rev. Lett. 77(13), 2670 2673 (1996)

6. J. Homola, S.S. Yee, G. Gauglitz, Surface plasmon resonance sensors: review. Sens. Actuators B, Chem. 54(1-2), 3-15 (1999)

7. P. Dawson, F. Defornel, J.P. Goudonnet, Imaging of surfaceplasmon propagation and edge interaction using a photon scanning tunneling microscope. Phys. Rev. Lett. 72(18), 2927-2930 (1994)

8. J.C. Weeber, J.R. Krenn, A. Dereux, B. Lamprecht, Y. Lacroute, J.P. Goudonnet, Near-field observation of surface plasmon polariton propagation on thin metal stripes. Phys. Rev. B 6404(4), $045411(2001)$
9. M.L.M. Balistreri, J.P. Korterik, L. Kuipers, N.F. van Hulst, Local observations of phase singularities in optical fields in waveguide structures. Phys. Rev. Lett. 85(2), 294-297 (2000)

10. A. Nesci, R. Dandliker, M. Salt, H.P. Herzig, Measuring amplitude and phase distribution of fields generated by gratings with subwavelength resolution. Opt. Commun. 205(4-6), 229-238 (2002)

11. E. Fluck, M. Hammer, A.M. Otter, J.P. Korterik, L. Kuipers, N.F van Hulst, Amplitude and phase evolution of optical fields inside periodic photonic structures. J. Lightwave Technol. 21(5), 13841393 (2003)

12. H.L. Offerhaus, B. van den Bergen, M. Escalante, F.B. Segerink, J.P. Korterik, N.F. van Hulst, Creating focused plasmons by noncollinear phasematching on functional gratings. Nano Lett. 5(11), 2144-2148 (2005)

13. J. Jose, F.B. Segerink, J.P. Korterik, H.L. Offerhaus, Near-field observation of spatial phase shifts associated with Goos-Hanschen and surface plasmon resonance effects. Opt. Express 16(3), 19581964 (2008)

14. E. Kretschmann, H. Raether, Radiative decay of nonradiative surface plasmons excited by light. Z. Naturforsch. A 23, 2135-2136 (1968)

15. U. Schroter, D. Heitmann, Grating couplers for surface plasmons excited on thin metal films in the Kretschmann-Raether configuration. Phys. Rev. B 60(7), 4992-4999 (1999)

16. R. Stierlin, R. Battig, P.D. Henchoz, H.P. Weber, Excess-noise suppression in a fiberoptic balanced heterodyne-detection system. Opt. Quantum Electron. 18(6), 445-454 (1986)

17. A.G.T. Ruiter, J.A. Veerman, K.O. van der Werf, N.F. van Hulst, Dynamic behavior of tuning fork shear-force feedback. Appl. Phys. Lett. 71(1), 28-30 (1997) 